\title{
Biomarkers and Echocardiography in the Postoperative Course of Pulmonary Resection Surgery
}

\author{
David Andaluz-Ojeda ${ }^{1}$, Francisco Gandía ${ }^{1}$, Jose Luis Duque ${ }^{2}$, Itziar Gómez ${ }^{3}$, Begona De Gregorio ${ }^{2}$, \\ Jose Alberto San Román ${ }^{3}$, Eduardo Villacorta ${ }^{3}$ \\ ${ }^{1}$ Department of Intensive Care Medicine, Hospital Clínico Universitario, Valladolid, Spain; ${ }^{2}$ Deparment of Thoracic Surgery, Hospi- \\ tal Clínico Universitario, Valladolid, Spain; ${ }^{3}$ Deparment of Cardiology, Hospital Clínico Universitario, Valladolid, Spain. \\ Email: davidandaluz78@yahoo.es
}

Received August $4^{\text {th }}, 2011$; revised August $28^{\text {th }}, 2011$; accepted September $7^{\text {th }}, 2011$.

\begin{abstract}
Background: The existence of different degrees of inflammatory reaction and right ventricular dysfunction after pulmonary resection is well known, though its detection and clinical relevance have not been sufficiently evaluated. The introduction of new biomarkers, particularly brain natriuretic peptide (BNP) and its prohormone (proBNP), together with dynamic imaging techniques such as echocardiography, could prove useful in assessing such response. The aim of this study is to describe the kinetic curve of these biomarkers during uncomplicated pulmonary resection surgery. Furthemore we have studied the right ventricular function by echocardiography in these patients. Methods: Demographic data, antecedents and certain perioperative parameters, as well as plasma proBNP, troponin $T$ and C-reactive protein $(C R P)$ levels, were recorded in 38 patients who underwent pneumonectomy $(n=13)$ and lobectomy $(n=25)$, before and 24, 48 and 72 hours after the operation. A transthoracic echocardiogram was carried out preoperatively and 72 hours after surgery. Results: ProBNP showed a significant increase 24 hours after surgery, with a maximum peak after 72 hours $(p<0.001)$. CRP likewise increased after 24 hours, with an inflammatory peak after 48 hours $(p<0.001)$. The magnitude of the increase in proBNP and CRP was independent of the extent of resection (pneumonectomy versus lobectomy). Echocardiography showed a slight yet statistically significant decrease in right ventricular function (determined by tricuspid annular plane systolic excursion, TAPSE) in the pneumonectomy subgroup $(p=0.01)$. Conclusion: The plasma proBNP elevation curve allows simple evaluation of the degree and time course of right ventricular dysfunction in the immediate postoperative period of uncomplicated pulmonary resection surgery. In stable patients, magnitude of proBNP elevation has no clinical repercussions, while slight echocardiographic changes are detectable only in pneumonectomized patients. Further studies are needed to evaluate the usefulness of abnormal proBNP elevations in predicting cardiorespiratory complications at patients bedside during the immediate postoperative period.
\end{abstract}

Keywords: Pneumonectomy, Lobectomy, Echocardiography, Right Ventricle, Natriuretic Peptides, C Reactive Protein, T Troponine, Biomarkers

\section{Introduction}

It is well known that extensive pulmonary resections are related to a number of postoperative alterations in right ventricular function. Decrease of the pulmonary vascular bed area after such surgery implies an increase in pulmonary artery pressure (PAP), which can lead to an increase in right ventricular (RV) afterload. As result, a series of hemodynamic changes and readjustments take place [1,2], though their time course during postoperative period and its possible usefulness in clinical practice have not been studied to date.
The recent introduction of new biological markers implicated in cardiorespiratory homeostasis, particularly atrial natriuretic peptide (ANP), brain natriuretic peptide (BNP) and BNP prohormone (proBNP), and their increasingly widespread use in routine clinical practice, have served to establish their diagnostic, prognostic and monitoring usefulness in patients with heart failure [3]. These peptides are synthesized by the myocardiocytes, and their production increases significantly in response to all factors that subject heart muscle to pressure and/or volume overload with subsequently increased ventricular 
wall stress [4].

The diagnostic and therapeutic potential of these natriuretic peptides in the context of left ventricular dysfunction (congestive heart failure or ischemia) has been extensively documented $[5,6]$. However, and in contrast to the extensive literature on natriuretic peptide and left ventricular dysfunction, fewer studies have analyzed right ventricular function. In this context, a number of recent studies have reported a significant increase in BNP or proBNP levels in disorders that imply RV overload, such as pulmonary thromboembolism $[7,8]$, chronic obstructive pulmonary disease (COPD) and cor pulmonale [9], or in pulmonary hypertension [10]. Little is known about the behavior of these molecules in the postoperative period of major pulmonary resection surgery.

The aim of this study was to determine the kinetic of certain biological markers in patients requiring thoracotomy and pulmonary resection, examining the perioperative time course of the serum levels of three biomarkers: proBNP, troponin $\mathrm{T}(\mathrm{TnT})$ and C-reactive protein (CRP). With the purpose of establishing the behavior of proBNP as a marker of right-side cardiac function, comparisons have been made of serum proBNP and the echocardiographic changes in right ventricular dynamics induced by surgical procedures. In addition, comparisons have been made of the possible differential characteristics between lobectomy and pneumonectomy patients.

\section{Material and Methods}

\subsection{Patients}

After obtaining institutional approval and the patients' consent, between October 2009 and May 2010, data on all consecutive patients who underwent major pulmonary resection surgery were collected. Video-assisted thoracic surgery were no considered. All patients with a history of ischemic heart disease, severe renal failure, cardiac arrhythmias, heart failure or decompensated arterial hypertension were excluded from the study. We also excluded those subjects who developed cardiological complications - fundamentally arrhythmias or ischemic events-in the course of the study, since these problems intrinsically imply proBNP elevation and echocardiographic alterations. A total of 38 patients scheduled for pulmonary resection surgery were finally included in the study. In all cases the preoperative workup comprised a physical examination, general laboratory tests, chest X-rays, 12-lead electrocardiography, spirometry and arterial blood gas determinations.

The following variables were recorded: a) demographic data and antecedents: sex, age, comorbidities and respiratory function tests; b) perioperative data: type of surgery, type of anesthesia, hemodynamic parameters, surgery and anesthesia times, blood losses, transfusion of blood products, and intraoperative complications; and c) complications during the immediate postoperative period, classified into three main groups: cardiological, infectious and surgical. Acute complications were taken to be those problems manifesting in the first 72 hours after surgery, during the period established for determination of the biological markers. During surgery, the patients were monitored through radial artery catheterization, and mechanical ventilation was carried out using a doublelumen orotracheal tube.

\subsection{Determinations}

Peripheral blood samples were collected from all patients at the following timepoints: preoperative control, in the Intensive Care Unit immediately after surgery, and again after 24, 48 and 72 hours. The samples were collected in pararalel by using tubes containing ethylenediaminetetraacetic acid (EDTA), immediataly cooled to $4^{\circ} \mathrm{C}$ and centrifuged. Determinations were made of plasma proBNP, CRP and TnT. Chemiluminescence techniques were used for the determination of proBNP and TnT (HELECSYS 2010 and Cobas e601; Hitachi, Japan, distributed by Roche), respectively, while turbidimetric latex immunoagglutination techniques were used to determine CRP (Cobas c311, Roche Diagnostics GmbH).

\subsection{Echocardiogram}

Echocardiography in all cases was carried out with two echocardiographic systems equipped with a 1.5 to $3 \mathrm{MHz}$ transducer (Vivid 3; General Electric; HDI 5000; ATL). Transthoracic echocardiography was performed preoperatively and again 72 hours after surgery. The explorations were made by an investigator blinded to the type of surgery involved, and to the patient biomarker levels. Those patients in which transthoracic echocardiographic images of sufficient quality could not be obtained were excluded from the study.

Left ventricular function was calculated using the Teichholz method in M-mode, or the Simpson method. Altered left ventricular function was defined by an ejection fraction (EF) of under 55\% [11].

Right ventricular function was evaluated by measuring tricuspid annular plane systolic excursion (TAPSE). The latter was defined as the difference in right ventricle base displacement or excursion during systole and diastole. Normal RV function was defined by TAPSE $>19 \mathrm{~mm}$ [12-15].

\subsection{Statistical Analyses}

Categorical variables are given as the absolute number and percentage, while continuous variables are given as the mean \pm standard deviation (SD) and/or median and 
interquartile range. The Shapiro-Wilk test was used to check the normal distribution of the quantitative variables. Naperian logarithmic transformation of the variables CRP and proBNP was used to secure normality.

The presence or absence of pneumonectomy was used to divide the patients into two groups, which were compared using the chi-squared test, with application of the Fisher exact test where necessary in the case of qualitative variables. Continuous variables were compared using the Student t-test.

The time course of proBNP and CRP was analyzed by repeated measures analysis of variance (ANOVA), using simple contrasting and the preoperative value of each variable as reference category.

The pre/postoperative course of the echocardiographic parameters was analyzed using the Student t-test for paired samples.

All data were included in an SPSS Inc database (Version 15.0, Chicago, Illinois). Statistical significance was accepted for $\mathrm{p}<0.05$.

\section{Results}

\subsection{Patients}

The mean age was 65.4 years, and $92.1 \%$ were males. Mean preoperative vital capacity (VC) was $3356.5 \mathrm{ml}$, with a forced expiratory volume in one second $\left(\mathrm{FEV}_{1}\right)$ of $2277.13 \mathrm{ml}$. A prior diagnosis of COPD had been established in $18.4 \%$ of the subjects, and $84.2 \%$ of them were smokers. In most cases (72.4\%) lung cancer was the indication for surgery.

Initially 28 lobectomies (including 4 bilobectomies) and 15 pneumonectomies were performed. Five patients (11.6\%) developed arrhythmias during the study period (atrial fibrillation in all cases), and were therefore excluded (3 lobectomies and 2 pneumonectomies) - the final series thus comprising 25 lobectomies and 13 pneumonectomies.

Table 1 separately compares the baseline clinical characteristics of the pneumonectomy versus the lobectomy patients. No significant differences were observed in any of the study parameters. Likewise, no statistically significant differences were observed between the two subgroups in terms of surgery time, anesthesia time or hemodynamic parameters (mean arterial pressure and central venous pressure) during surgery. Only one patient required blood product transfusions during study period. There were no intraoperative deaths (Table 2).

Regarding late complications, two cases of infection were documented (4.6\%), one of the upper airway and another corresponding to severe pneumonia and late sepsis leading to the death of the patient 21 days after surgery. Four episodes of bronchopleural fistula were recorded 12, 17, 35 and 150 days after surgery $(9.3 \%$ of all patients).

The severity prognostic scores obtained with the APACHE II 24 hours after surgery showed no differences between the both subgroups $(6.9 \pm 3.4$ versus $7.4 \pm$ 2.2; $\mathrm{p}=0.601$ ).

\subsection{Biological Markers}

ProBNP:

Statistically significant increase in plasma proBNP levels was recorded in all patients 24 hours after surgery, with a maximum elevation after 72 hours. This same behavior was observed on considering proBNP levels separately in each subgroup. However, there were no statistically significant differences on comparing serum proBNP in the pneumonectomy patients with those in the lobectomy group (Figure 1, Table 3).

Troponin T:

Table 1. Baseline patient characteristics.

\begin{tabular}{l|c|c|c}
\hline \multicolumn{1}{c|}{ Characteristics } & $\begin{array}{c}\text { Lobectomy } \\
\mathrm{n}=25\end{array}$ & $\begin{array}{c}\text { Pneumonectomy } \\
\mathrm{n}=13\end{array}$ & $\mathbf{p}$ \\
\hline Age & $63.4 \pm 1.3$ & $64.3 \pm 8$ & 0.809 \\
Male/female & $22 / 3$ & $13 / 0$ & 0.538 \\
Smoking & $21(84 \%)$ & $11(84.6 \%)$ & 0.999 \\
$\mathrm{COPD}$ & $4(16 \%)$ & $3(23.1 \%)$ & 0.672 \\
$\mathrm{FEV}$ & $(\mathrm{l})$ & $2.3 \pm 0.8$ & 0.488 \\
$\mathrm{FEV}_{1} \%(\mathrm{l})$ & $2.4 \pm 0.6$ & $83.5 \pm 20.5$ & 0.346 \\
$\mathrm{VC}(\mathrm{l})$ & $89.5 \pm 17.2$ & $3.4 \pm 0.7$ & 0.666 \\
$\mathrm{VC} \%$ & $3.3 \pm 0.9$ & $96.4 \pm 20$ & 0.876 \\
Baseline $\mathrm{SatO}_{2}$ & $97.5 \pm 20$ & $96.1 \pm 1$ & 0.674 \\
\hline
\end{tabular}

Quantitative variables are given as the mean \pm standard deviation. No significant differences were found between the two groups for any of the parameters considered. $\mathrm{FEV}_{1}=$ forced expiratory volume in one second; $\mathrm{FEV}_{1} \%$ = predicted percentage $\mathrm{FEV}$; $\mathrm{VC}=$ vital capacity; $\mathrm{VC} \%=$ predicted percentage VC; COPD = Cronic obstructive pulmonary disease. 
Table 2. Perioperative patient characteristics.

\begin{tabular}{l|c|c|c}
\hline \multicolumn{1}{c|}{ Characteristics } & $\begin{array}{c}\text { Lobectomy } \\
\mathrm{n}=25\end{array}$ & $\begin{array}{c}\text { Pneumonectomy } \\
\mathrm{n}=13\end{array}$ \\
\hline Surgery time (min) & $181.4 \pm 5$ & $191.1 \pm 36.4$ & 0.52 \\
Anesthesia time (min) & $232.5 \pm 59.2$ & $240.8 \pm 43.9$ & 0.67 \\
Epidural & $7(29.2 \%)$ & $6(46.2 \%)$ & 0.47 \\
Paravertebral & $15(62.5 \%)$ & $7(53.8 \%)$ & 0.60 \\
$\mathrm{MAP}(\mathrm{mmHg})$ & $85 \pm 15.9$ & $92.7 \pm 18.9$ & 0.24 \\
$\mathrm{CVP}(\mathrm{cms} \mathrm{H} \mathrm{O})$ & $6.2 \pm 3.1$ & $5.5 \pm 4.3$ & 0.32 \\
$\mathrm{SatvO}_{2}$ & $76.7 \pm 8.2$ & $72 \pm 16.3$ & 0.35 \\
$\mathrm{PaO}_{2} / \mathrm{FiO}_{2}$ & $308.4 \pm 95$ & $272.4 \pm 96.4$ & 0.35 \\
$\mathrm{Hb}_{\text {post }}$ & $11.4 \pm 0.3$ & $11.2 \pm 0.4$ & 0.42 \\
Fluid $_{\text {post }}(\mathrm{ml})$ & $1975 \pm 340$ & $1435 \pm 218$ & 0.12 \\
\hline
\end{tabular}

Quantitative variables are given as the mean \pm standard deviation. No significant differences were found between the two groups for any of the parameters considered. $\mathrm{MAP}=$ mean arterial pressure; $\mathrm{SatvO}_{2}=$ venous oxygen saturation; $\mathrm{PaO}_{2} / \mathrm{FiO}_{2}=$ arterial $\mathrm{O}_{2}$ pressure/inspiratory $\mathrm{O}_{2}$ fraction; $\mathrm{Hb}_{\text {post }}=$ serum hemoglobin after surgery; Fluid post = total fluid administration the first 24 hours after the surgery.
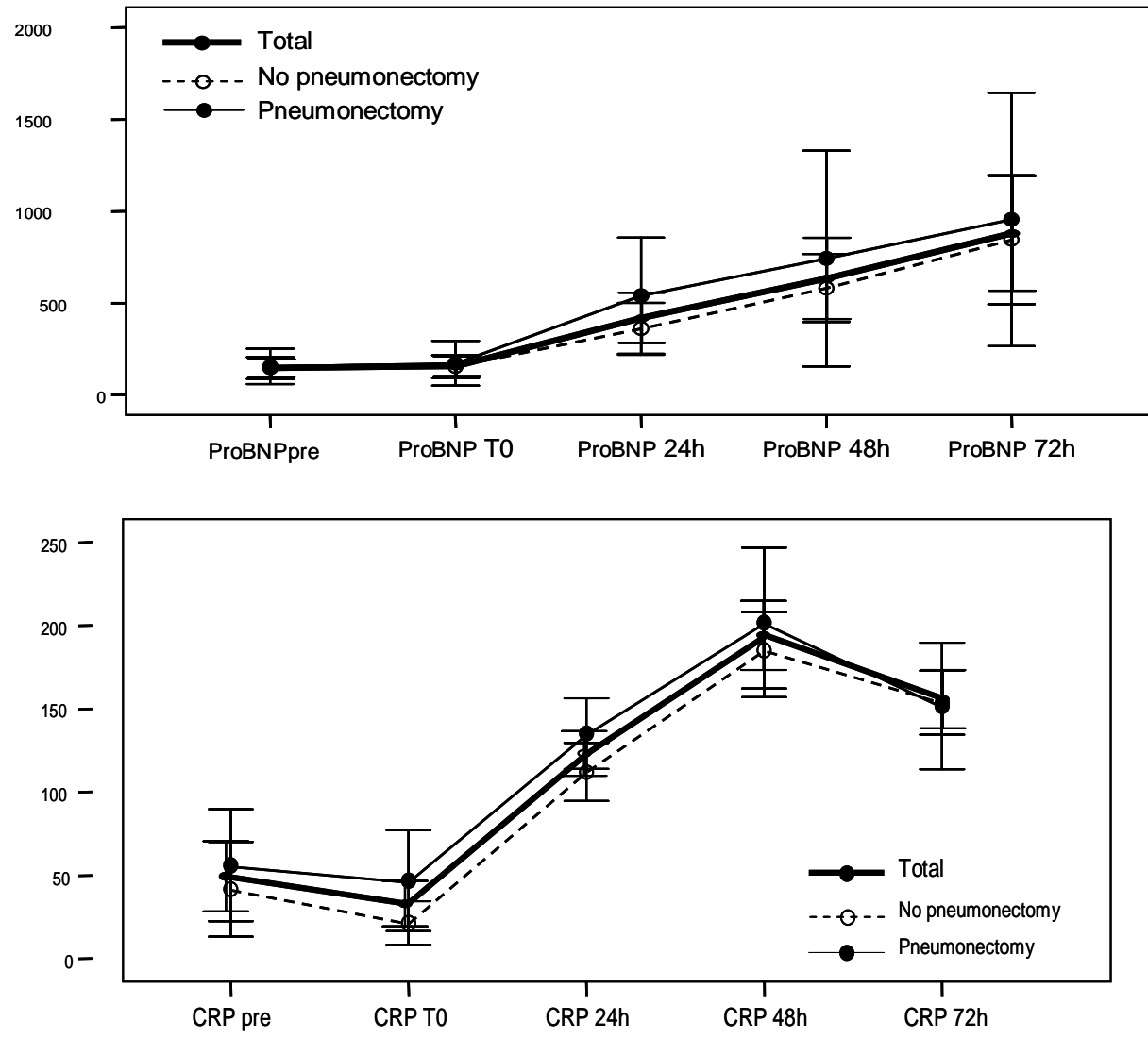

(a)

Figure 1. (a)Serum proBNP levels (pg/ml) at baseline (pre), immediately after surgery (T0), and after 24,48 and 72 hours in the global patients and in the pneumonectomy and non-pneumonectomy subgroups. The values are given as the mean \pm standard deviation, with the corresponding $95 \%$ confidence interval $(95 \% \mathrm{CI})$. In all cases a significant increase in proBNP is observed starting from 24 hours after surgery, with a maximum elevation after 72 hours $(p<0.001)$. (b) Serum CRP levels at baseline (pre), immediately after surgery (TO), and after 24, 48 and 72 hours in the global patients and in the pneumonectomy and non-pneumonectomy subgroups. The values are given as the mean \pm standard deviation, with the corresponding $95 \%$ confidence interval $(95 \% \mathrm{CI})$. In all cases a significant increase in CRP is observed starting from 24 hours after surgery, with a maximum elevation after 48 hours $(p<0.001)$. 
No TnT elevations were observed, except in two patients, and in both such increases did not reach clinical significance. None of the study subjects experienced cardiological or ischemic complications during the study period.

CRP:

A statistically significant increase in CRP was recorded starting from 24 hours after surgery, with a maximum elevation after 48 hours. There were no differences on comparing CRP levels in both subgroups of patients (Figure 1, Table 3).

\subsection{Echocardiography}

Transthoracic echocardiography was performed preoperatively in 28 patients (74\%) and again 72 hours after surgery in 27 subjects (71\%).

The echocardiographic findings are commented in Table 4. Preoperative values corresponding to biventricular function were within normal limits both, globally and in the two patient subgroups. However, in the postoperative period, a decrease was noted in right ventricular function (quantified by TAPSE) compared with preoperative function $(22.98 \pm 3.71$ versus $21.18 \pm 3.27$; $\mathrm{p}=$ $0.005)$, in pneumonectomized patients $(23.7 \pm 2.8$ versus $20.9 \pm 0.2 .83 ; p=0.011$ ). There were no such differences in right ventricular function in the lobectomy subgroup ( $22.5 \pm 4.1$ versus $21.3 \pm 3.6$; $p=0.131)$. Left ventricle ejection fraction was not affected by surgery in either the global patient series $(70.9 \pm 5.83$ versus $68.35 \pm 5.93$; $\mathrm{p}=$ 0.208 ) or in the two subgroups.

Table 3. Time comparison of proBNP and CRP levels between lobectomy and pneumonectomy patients.

\begin{tabular}{ccccc}
\hline & $\begin{array}{c}\text { Total } \\
(\mathbf{n}=\mathbf{3 8})\end{array}$ & $\begin{array}{c}\text { No pneumonectomy } \\
\mathbf{( n = 2 5 )}\end{array}$ & $\begin{array}{c}\text { Pneumonectomy } \\
(\mathbf{n}=\mathbf{1 3})\end{array}$ & P (between groups) \\
\hline ProBNPpre (pg/ml) & $4.34 \pm 1.14$ & $4.34 \pm 1.23$ & $4.31 \pm 0.97$ & 0.941 \\
ProBNPT0 (pg/ml) & $4.44 \pm 1.01$ & $4.48 \pm 1.04$ & $4.35 \pm 0.96$ & 0.721 \\
ProBNP24h (pg/ml) & $5.60 \pm 0.86 *$ & $5.58 \pm 0.88 *$ & $5.64 \pm 0.85 *$ & 0.845 \\
ProBNP48h (pg/ml) & $5.93 \pm 0.81 *$ & $6.03 \pm 0.69 *$ & $5.74 \pm 1.01 *$ & 0.310 \\
ProBNP72h (pg/ml) & $5.96 \pm 1.05 *$ & $6.00 \pm 1.05 *$ & $5.87 \pm 1.10 *$ & 0.723 \\
CRPpre (mg/l) & $2.63 \pm 1.91$ & $2.36 \pm 2.02$ & $3.12 \pm 1.68$ & 0.257 \\
CRPT0 (mg/l) & $2.35 \pm 1.79$ & $1.94 \pm 1.88$ & $3.06 \pm 1.39$ & 0.070 \\
CRP24h (mg/l) & $4.68 \pm 0.51 *$ & $4.59 \pm 0.58 *$ & $4.83 \pm 0.35 *$ & 0.193 \\
CRP48h (mg/l) & $5.18 \pm 0.36 *$ & $5.14 \pm 0.33 *$ & $5.26 \pm 0.42 *$ & 0.361 \\
CRP72h (mg/l) & $4.97 \pm 0.34 *$ & $4.96 \pm 0.34 *$ & $4.99 \pm 0.37 *$ & 0.752 \\
\hline
\end{tabular}

The values are given as the mean \pm standard deviation of the Naperian logarithm of the proBNP and CRP levels. No significant differences in proBNP and CRP values were seen between both subgroups. ProBNPpre = preoperative proBNP values; proBNPT0 = proBNP values immediately after surgery; proBNP 24, 48 and $72 \mathrm{~h}=$ proBNP values 24, 48 and 72 hours after surgery, respectively. CRPpre = preoperative CRP values; CRPT0 = CRP values immediately after surgery; CRP 24, 48 and $72 \mathrm{~h}=\mathrm{CRP}$ values 24,48 and 72 hours after surgery, respectively. * p < 0.01 versus prior to surgery (proBNPpre or CRPpre respectivily).

Table 4. Echocardiographic results.

\begin{tabular}{|c|c|c|c|}
\hline TAPSE (mm) & Preoperative & Postoperative & $\mathbf{p}$ \\
\hline Global patients & $22.98 \pm 3.71$ & $21.18 \pm 3.27$ & 0.005 \\
\hline Pneumonectomy & $23.75 \pm 2.80$ & $20.93 \pm 2.83$ & 0.01 \\
\hline Lobectomy & $22.53 \pm 4.16$ & $21.33 \pm 3.58$ & 0.13 \\
\hline \multicolumn{4}{|l|}{ LVF (\%) } \\
\hline Global patients & $70.94 \pm 5.83$ & $68.35 \pm 5.93$ & 0.20 \\
\hline Pneumonectomy & $71.67 \pm 5$ & $67 \pm 7.48$ & 0.21 \\
\hline Lobectomy & $70.54 \pm 6.40$ & $69.09 \pm 5.14$ & 0.57 \\
\hline \multicolumn{4}{|l|}{ LVEDV (ml) } \\
\hline Global patients & $64.67 \pm 14.49$ & $67.25 \pm 16.51$ & 0.74 \\
\hline Pneumonectomy & $51.33 \pm 8.50$ & $84.0 \pm 24.55$ & 0.22 \\
\hline Lobectomy & $69.11 \pm 13.47$ & $61.67 \pm 9.15$ & 0.23 \\
\hline
\end{tabular}

Quantitative variables are given as the mean \pm standard deviation. LVF = left ventricular function; TAPSE = tricuspid annular plane systolic excursion; LVEDV = left ventricle end-diastolic volume. 


\section{Comment}

The present study describes the kinetics of proBNP release during the immediate postoperative period of major pulmonary resection surgery, showing an increase in the serum concentration of the molecule noted from 24 hours after surgery, with a maximum increase after 72 hours. This coincides with the observations of one of two published studies on this subject, in which Hoksch et al. [16] likewise observed an increase (in this case of BNP) from 24 hours after surgery. However, the other study, published by Tayama et al. [17], found only the pneumonectomized patients to present a significant increase in BNP 72 hours after surgery.

The physiological action of proBNP results in an adaptive response to cardiovascular stress. This limits myocardial hypertrophy causing peripheral vasodilatation, with a modest negative inotropic effect and inhibition of renal renin and angiotensin production. The main clinical benefit is fundamentally the lowered preload secondary to increased sodium urine excretion and diuresis [18]. The molecule is a potent predictor of ventricular dysfunction that even contributes prognostic significance [19].

Our results showed a very significant increase in proBNP 24 hours after surgery, with a maximum elevation after 48 - 72 hours. The release of the molecule would be a consequence of the adaptive response of the right ventricle to the reduction in pulmonary capillary bed after surgery, and the subsequent increase in end-diastolic pressure in the right cavities of the heart.

In contrast to other authors, we observed no significant differences in proBNP levels comparing pneumonectomy with lobectomy group. However, Tayama et al. [16] did report differences between both groups, with higher BNP and ANP levels and an increase in PVR and PAP in the pneumonectomized patients. This is to be expected, since the lesser capillary cross-sectional area in pneumonectomized subjects. However, the fact that we excluded patients with previous heart disease or those who developed acute cardiological complications in the postoperative period (fundamentally arrhythmias) can explain the absence of differences in the values of the mentioned molecule. Since the included patients had a healthy ventricle, the differences in PVR between the lobectomy group and the pneumonectomy series might be not large enough to yield a significant difference in proBNP values between both groups.

The effect of pulmonary resection upon the functional dynamics of the right ventricle remains no clear. Some authors have reported a slight postoperative decrease in RV function [20-22], while others have observed no changes [23]. In our patients the postoperative variations in TAPSE indicated a thin decrease in RV function during the immediate postoperative period, but only in the pneumonectomy subgroup. Nevertheless, despite the observation of diminished RV function, the reduction is minimal and of scant clinical relevance. In fact, values recorded during the postoperative interval were within normal limits, and no hemodynamic repercussions were observed.

As regards troponin $\mathrm{T}$, literature makes no mention of the usefulness of this molecule in the postoperative period of major pulmonary resection surgery, and no descriptions can be found of TnT release kinetics in such situations. In our study we recorded no increase in TnT levels. It therefore can be concluded that rutinary TnT monitorization offers no useful information, and only seems to be indicated in those cases where ischemic heart disease is suspected.

There are not many references to CRP in relation to major pulmonary resection surgery. Franke et al. [24] reported a greater increase in CRP and other inflammatory markers (procalcitonin, IL-6 and lipoprotein-binding protein (LBP)) in partial resections versus pneumonectomy, observing an inflammatory peak 24 hours after the operation. The authors attributed these findings to ischemia-reperfusion phenomena and reventilation occurring during partial resection in the operated lung. Of note, however, is the fact that Franke et al. did not differentiate those patients who may have suffered complications of any kind (including infections) - this being a clear potential source of bias. We observed no such differences and CRP levels were seen to be similar in both patient groups, with significant elevation 24 hours after surgery and a significant maximum elevation after 48 hours.

The immune response to surgical trauma is complex. Surgery stimulates activation of the Mononclear-phagocyte cell system. These cells transform the initially local inflammatory phenomenon into a systemic inflammatory response through the release of inflammatory cytokines such as Il-6 or IL-8, which in turn increase the genetic expression of acute-phase reactants such as LBP or CRP at hepatocyte level [25,26].

Although the reperfusion of lung tissue traumatized by ischemia and by the surgical operation itself, together with reventilation following selective single-lung ventilation during partial resection surgery, could induce more-or-less intense inflammation, we consider that the enhanced flow received by the healthy lung after pneumonectomy, and which results in increased PVR and PAP values, could also cause damage and inflammation at pulmonary capillary endothelial level. Thus, both types of surgery would induce inflammatory phenomena through different mechanisms.

Our study has some limitations. Probably the most 
important is the small sample size. Another limitation is represented by the fact that maximum proBNP values were recorded on the last day of sample collection (72 hours after surgery) - thus raising the question of Whethe proBNP levels could have increased beyond this timepoint. Moreover, an unique echocardiogram was performed during the postoperative period, making it difficult to correlate it with successive changes in biological marker levels. Additional studies probably would be needed, involving a larger number of patients, and extending both, the hormone measurement period and the number of echocardiograms performed during the postoperative period.

In conclusion, we consider that the present study contributes to the understanding of the postoperative period of uncomplicated pulmonary resection surgery, analyzing the use of imaging techniques in cardiopulmonary hemodynamic physiopathology and the release of certain selected biological markers. Troponin $\mathrm{T}$ levels are not modified in uncomplicated chest surgery. As regards C-reactive protein, showed an increase 24 hours after surgery, with a maximum elevation after 48 hours regardless the type of surgery involved.

The proBNP release kinetics showed a significant increase 24 hours after surgery with a maximum elevation after 72 hours. No differences were recorded in terms of the extent of pulmonary resection. Likewise, echocardiography revealed a slight decrease in right ventricular function in the pneumonectomy subgroup, though the values of RV function remained within normal limits. Finally, it could be concluded that in absence of cardiological complications, proBNP levels above the mean reported values, or with elevation kinetics different from those described, could indicate the presence of RV dysfunction in the postoperative period of major pulmonary resections. To prove this assertion new studies should be designed to establish the clinical usefulness and correlation of natriuretic peptides as a simple marker of the severity of different postoperative cardiorespiratory complications.

\section{REFERENCES}

[1] C. E. Reed, H. E. Dorman and F. G. Spinale, "Effect of Pulmonary Resection on Right Ventricular Function," The Annals Thoracic Surgery, Vol. 53, 1992, pp. 578-582. doi:10.1016/0003-4975(92)90314-T

[2] C. E. Reed, H. E. Dorman and F. G. Spinale, “Assesment of Rentricular Contractile Performance after Pulmonary Resection," The Society of Thoracic Surgeons, Vol. 56, 1993, pp. 426-432.

[3] M. R. Cowie, A. D. Struthers, D. A. Wood, et al., "Value of Natriuretic Peptides in Assessment of Patients with Possiblen New Heart Failure in Primary Care,” Lancet
Vol. 350, No. 9088, 1997, pp. 1347-1351.

[4] L. King and M. R. Wilkins, "Natriuretic Peptides Receptors and the Heart," Heart, Vol. 87, NO. 4, 2002, pp. 314-315. doi:10.1136/heart.87.4.314

[5] A. Maisel, P. Krishnaswamy, R. Nowak, et al., "Rapid Measurement of B-Type Natriuretic Peptide in the Emergency Diagnosis of Heart Failure," The New England Journal Medicine, Vol. 347, 2002, pp. 161-167. doi:10.1056/NEJMoa020233

[6] J. A. De Lemos, D. K. Mc Guire and M. H. Drazner, "BType Natriuretic Peptide in Cardiovascular Disease," Lancet, Vol. 362, 2003, pp. 316-322.

[7] M. Ten Wolde, Tulevski II, J. W. Mulder, et al., "Brain Natriuretic Peptide as a Predictor of Adverse Outcome in Patients with Pulmonary Embolism," Circulation, Vol. 117, 2003, pp. 2082-2084. doi:10.1161/01.CIR.0000070020.79932.DB

[8] N. Kucher, G. Printzen and S. Z. Goldhaber, "Pronostic Role of Brain Natriuretic Peptide in Acute Pulmonary Embolism," Circulation, Vol. 107, 2003, pp. 2545-2547.

[9] M. Bando, Y. Isii, Y. Sugiyama, et al., "Elevated Plasma Brain Natriuretic Levels in Chronic Respiratory Failure with Cor pulmonale,” Respiratory Medicine, Vol. 93,No. 7, 1999, pp. 507-514. doi:10.1016/S0954-6111(99)90094-X

[10] N. Nagaya, T. Nikishimi, M. Uematse, et al., "Plasma Brain Natriuretic Peptide as a Prognostic Indicator in Patients with Primary Pulmonary Hypertension," Circulation, Vol. 102, 2000, pp. 865-870.

[11] M. A. Quinones, C. M. Otto, M. Stoddard, A. Wggoner and W. A. Zoghbi, "Recommendations for Quantification of Doppler Echocardiography: A Report from the Doppler Quantification Task Force of the Nomenclature and Standards Committee of the American Society of Echocardiography," Journal of American Society Echocardiogrdiography, Vol. 15, No. 2, 2002, pp. 167-184.

[12] S. Kaul, C. Tei, J. M. Hopkins and P. M. Shan, "Assessment of Right Ventricular Function Using Two Dimensional Echocardiography,” American Heart Journal, Vol. 107, No. 3, 1984, pp. 526-531. doi:10.1016/0002-8703(84)90095-4

[13] J. Meluzin, L. Spinarova, J. Bakala, J. Toman, J. Krejci, P. Hude, T. Kara and M. Soucek, "Pulsed Doppler Tissue Imaging of the Velocity of Tricuspid Annular Systolic Motion. A New, Rapid, and Non-Invasive Method of Evaluating Right Ventricular Systolic Function,” European Heart Journal, Vol. 22, No. 4, 2001, pp. 280-282. doi:10.1053/euhj.2000.2296

[14] K. Miyatake, M. Yamgishi, N. Tanaka, M. Uematsu, N. Yamazaki, Y. Mine, A. Sano and M. Hirama, "New Method for Evaluating Left Ventricular Wall Motion by Color-Coded Tissue Doppler Imaging: In Vitro and In vivo Studies,” Journal American College Cardiology, Vol. 25, No. 3, 1995, pp. 717-724. doi:10.1016/0735-1097(94)00421-L

[15] O. M. Ueti, E. E. Camargo, A. Ueti Ade, E. C. de Lima-Filho and E. A. Nogueira, "Assessment of Right 
Ventricular Function with Doppler Echocardiographic Indices Derived from Tricuspid Annular Motion: Comparison with Radionuclide Angiography,” Heart, Vol. 88, No. 3, 2002, pp. 244-248.

[16] B. Hoksch, R. Hahner and R. A. Schmid, "Procalcitonin and Brain Natriuretic Peptide as Parameters in the Postoperative Course of Patients with Major Pulmonary Resection," Interact Cardiovasc Thoracic Surgery, Vol. 6, 2007, pp. 155-159. doi:10.1510/icvts.2006.143073

[17] K. Tayama, S. Takamori, M. Mitsuoka, et al., "Natriuretic Peptides after Pulmonary Resection,” The Annals Thoracic Surgery, Vol. 73, No. 5, 2002, pp. 1582-1586. doi:10.1016/S0003-4975(02)03417-3

[18] S. P. Souza, M. Davis and G. F. Baxter, "Autocrine and Paracrine Actions of Natriuretic Peptides in the Heart," Pharmacology Therapeutics, Vol. 101, No. 2, 2004, pp. 113-129. doi:10.1016/j.pharmthera.2003.11.001

[19] J. Koglin, S. Pehlivanli, M. Schwaiblmair, M. Voseger, P. Cremer and W. vonScheidt, "Role of Brain Natriuretic Peptide in Risk Stratification of Patients with Congestive Heart Failure," Journal American College Cardiology, Vol. 38, 2001, pp. 1934-1941. doi:10.1016/S0735-1097(01)01672-2

[20] C. E. Reed, F. G. Spinale and F. A. Crawford Jr., "Effect of Pulmonary Resection on Right Ventricular Function," The Annals Thoracic Surgery, Vol. 53, No. 4, 1992, pp. 578-582. doi:10.1016/0003-4975(92)90314-T

[21] C. E. Reed, B. H. Dorman and F. G. Spinale, "Mechanisms of Right Ventricular Dysfunction after Pulmonary Resection,” The Annals Thoracic Surgery, Vol. 62, No. 1, 1996, pp. 225-231. doi:10.1016/0003-4975(96)00258-5

[22] J. Kowalewski, M. Brocki, T. Dryjanski, K. Kapron and S. Barcikowski, "Right Ventricular Morphology and Function after Pulmonary Resection," European Journal of Cardio-Thoracic Surgery, Vol. 15, No. 1, 1999, pp. 444-448. doi:10.1016/S1010-7940(99)00032-9

[23] D. Amar, M. E. Burt, N. Roichaster, R. A. Reinsel, R. J. Ginsberg and R. S. Wilson, "Value of Perioperative Doppler Echocardiography in Patients Undergoing Major Lung Resection,” The Annals Thoracic Surgery, Vol. 61, 1996, pp. 516-520. doi:10.1016/0003-4975(95)00939-6

[24] A. Franke, W. Lante, S. Kupser, H. P. Becker, C. Weinhold, A. Markewitz, "Procalcitonin Levels after Different Types of Conventional Thoracic Surgery,” Thoracic Cardiovasc Surgery, Vol. 56, No. 1, 2008, pp. 46-50. doi:10.1055/s-2007-989250

[25] S. Wan, J. L. LeClerc and J. L. Vincent, “Cytokine Responses to Cardiopulmonary Bypass: Lessons Learned from Cardiac Transplantation," The Annals Thoracic Surgery, Vol. 63, 1997, pp. 269-276. doi:10.1016/S0003-4975(96)00931-9

[26] B. J. Grube, C. G. Cochane, R. D. Ye, et al., "Lipopolysaccharide Binding Protein Expression in Primary Human Hepatocytes and HepG2 Hepatoma Cells,” The Journal of Biological Chemistry, Vol. 269, 1994, pp. 8477-8482. 